\title{
Sitzung vom 26. October 1903.
}

Vorsitzender: Hr. C. Liebermann, Vicepräsident.

Das Protocoll der letzten Sitzung wird genehmigt.

Der Vorsitzende theilt mit, dass von dem Kaiserlich Russischen Finanzministerium, und zwar von der Hanptverwaltung der indirecten Steuern und des Branntweinmonopols, ein Preis von 50000 Rubeln für die Auffindung eines neuen Denaturirungsmittels für Spiritus ausgesetzt ist. An der Concurrenz um diesen Preis können sowohl russische Unterthanen wie auch Ansländer theilnehmen. Die näheren Bedingungen wollen unsere Mitglieder aus einer ausführlichen Mittheilung der genannten Behörde entnehmen, welche dem am 7. Norember erscheinenden Hefte 14 der $»$ Berichtes beigegeben wird.

Als ausserordentliche Mitglieder sind aufgenommen:

Hr. Ritsema, Dr. J.C., Haarlem; Hr. Milrog, Dr. J. A., Belfast;

"Silbermann, Dr. K., Augs- "Reichard, P., Berlin; burg;

Frl. Reimer, M., New York;

Hr. Siebert, Dr. C., Marburg;

D Albert, K., Wiesbaden;

- Kldiaschwili, Dr. A., Odessa;

¿Kohen, Dr. W., Hildesheim; » Thonet, R., Berlin.

Als ausserordentliche Mitglieder werden vorgeschlagen die HHrn.:

Steven, A. B., Yorkshire College, Leeds (durch A. G. Green und A. G. Perkin);

Frick e, Prof. Dr. K., Oberlehrer der Oberrealschule, Herderstrasse 62, Bremen (durch B. Tollens und A. Faust);

Rona, Dr. Peter, Grolmannstr. 58, Charlottenburg (durch R. Meyer und J. Biehringer);

B edford, Fred, Gr. Steinstr. 39, Halle a. S. (durch E. Erdmann und G. Baumert);

Berichte d. D. chem. Gesellschaft. Jahrg. XXXVI. 
Thode, Dr.: Ing. Carlos, Physiologisches Institut, Bonn (durch H. Pauly und W. Walter);

Lauder, Alexander, B. Sc., University College of North Wales, Bangor, London (durch K. J. P. Ortou und P. Jacobson).

Für die Bibliotbek sind als Geschenke eingegangen:

1711. Posner, Th. Lehrbuch der sgnthetisehen Methoden der organischen Chemie. Leipzig 1903.

1712. Söderbaum, H. G. Jac. Berzelius. Reseanteckninger. Stockholm 1903.

106. Jahresbericht über die Fortschritte der Chemie und verwandter Theile anderer Wissenschaften, begründet von J. Liebig und H. Kopp; herausgegeben von G. Bodlander und W. Kerp. Für 1898, Heft 2 und 3. Braunschweig 1903.
Der Vorsitzende:
Der Scbriftfübrer:
C. Liebermann.
W. Will.

\section{Mittheilungen.}

\section{J. v. Braun: Ueber eine neue gefärbte Klasse von Dithiourethanen.}

[Mittheilung aus dem Chemischen Institut der Universität Göttingen.]

(Eingegangen am 2. October 1903.)

In der vor einiger Zeit in Gemeinschaft mit F. Stechele') mitgetheilten Untersuchung über Thiuramsulfide worde hervorgeboben, dass diese Verbindungen $\mathrm{S}\left(\mathrm{CS} . \mathrm{NR}_{2}\right)_{2}$ sich durch intensive Gelbfärbung auszeichnen. Angesichts der Thatsache, dass eine lebhafte gelbe Farbe anch den Thiocarbaminoxyden $\mathrm{O}\left(\mathrm{CS} . \mathrm{NR}_{2}\right)_{2}$ und den Dithiobiureten RN $\left(\mathrm{CS} . \mathrm{NR}_{2}\right)_{2}$ znkommt, liegt der Schluss nahe, dass in den Thiuramsulfiden nicht das die beiden Thiocarbaminreste verbindende Schwefelatom für die Färbung maassgebend ist, sondern dass in allen drei Körperklassen die Färbung wesentlich durch die Thiocarbaminreste bedingt wird. Berücksichtigt man nun weiter, dass es andere Körperklassen mit demselben Rest.CS.NR giebt [Dithiourethane RS.CS.NR,

1) Diese Berichte 36, 2275 [1903]. 